\title{
Espessura do músculo adutor do polegar: um método rápido e confiável na avaliação nutricional de pacientes cirúrgicos
}

\section{Adductor pollicis muscle thickness: a fast and reliable method for nutritional assessment in surgical patients}

\author{
Rosalia Bragagnolo"; Fernanda Stephan Caporossi ${ }^{1}$; Diana Borges Dock-Nascimento²; José Eduardo de Aguilar- \\ NASCIMENTO, TCBC-MT ${ }^{3}$
}

\section{RE S U M O}

\begin{abstract}
Objetivo: Determinar se a medida da espessura do músculo adutor do polegar é confiável para avaliação nutricional de pacientes cirúrgicos e se correlaciona bem com outros parâmetros antropométricos, bioquímicos e clínicos. Métodos: Estudo de corte transversal, com avaliação de 87 pacientes candidatos à procedimento cirúrgico de grande porte no trato gastrointestinal. Eles foram submetidos à avaliação nutricional através da avaliação subjetiva global, antropometria (circunferência do braço, prega cutânea triciptal, e circunferência muscular do braço), albumina sérica, linfocitometria e pela medida da espessura do músculo adutor do polegar em ambas as mãos. Resultados: A média da espessura da mão dominante (direita) foi de 12,64 $\pm 3,19 \mathrm{~mm}$ e da mão não dominante $12,23 \pm 2,9 \mathrm{~mm}$. Para desnutrição, a sensibilidade da espessura do músculo adutor do polegar direita foi de $72,37 \%$ e da esquerda de $77,33 \%$ para os pontos de cortes encontrados com a curva Receiver Operating Characteristic $(13,4$ e 13,1 $\mathrm{mm}$ respectivamente). Para ambas as mãos a especificidade foi de $100 \%$. Encontrou-se correlação significativa $(p<0,01)$ da espessura do músculo adutor do polegar com todas as outras técnicas antropométricas de avaliação nutricional. Os seus valores médios nos pacientes eutróficos segundo a avaliação subjetiva global foram significativamente maiores $(\mathrm{P}<0.001)$ que dos desnutridos leves, e estes também maiores $(P<0,05)$ que os desnutridos graves (avaliação subjetiva global-C). Conclusão: a espessura do músculo adutor do polegar é um método de fácil execução, baixo custo, confiável e transmite segurança na avaliação do estado nutricional, podendo ser usado na prática clínica em pacientes cirúrgicos.
\end{abstract}

Descritores: Avaliação nutricional. Cirurgia. Desnutrição. Antropometria.

\section{INTRODUÇÃO}

Es studos mostram que a prevalência da desnutrição varia de 30\% a 50\% em pacientes cirúrgicos ${ }^{1,2}$. Além disso, a desnutrição hospitalar tem sido associada a maior tempo de internação, ao maior custo e ao aumento de complicações e mortalidade ${ }^{3}$. O gasto com pacientes desnutridos aumenta em cerca de $60,5 \%{ }^{4}$. Diante da reconhecida influência do estado nutricional sobre a evolução clínica de pacientes candidatos a procedimento cirúrgicos, todo esforço deve ser tomado para reconhecer e identificar pacientes portadores de desnutrição ou em risco de desenvolvêla. O objetivo é iniciar precocemente terapia nutricional pré-operatória e, assim, favorecer a recuperação do paciente no pós-operatório ${ }^{5}$.

O estado nutricional de pacientes hospitalizados pode ser avaliado por vários métodos dentre os quais se incluem levantamento de dados dietéticos, antropométricos, bioquímicos, imunológicos, história clínica e exame físico que permite estabelecer o diagnóstico nutricional ${ }^{6}$. Uma nova técnica para avaliar o compartimento muscular é a avaliação da espessura do músculo adutor do polegar (EMAP) por ser simples, não invasiva, rápida e de baixo Custo ${ }^{7}$. Alguns poucos autores têm padronizado medidas da EMAP para indivíduos saudáveis e pacientes hospitalizados $^{8-10}$, mas a literatura ainda é deficiente e há pouca informação quanto ao uso desta técnica de avaliação nutricional de pacientes cirúrgicos.

Este estudo tem como objetivo determinar se a medida da EMAP é confiável para avaliação nutricional de pacientes cirúrgicos e se ela correlaciona bem com outros parâmetros antropométricos, bioquímicos e clínicos.

\section{MÉTODOS}

Este trabalho foi aprovado pelo Comitê de Ética do Hospital Universitário Julio Muller da Universidade Federal do Mato Grosso, Cuiabá, MT, Brasil e os pacientes foram avaliados após consentimento livre e esclarecido. Foi

Trabalho realizado pela Equipe Multidisciplinar do Hospital Universitário Julio Muller, Universidade Federal de Mato Grosso, Cuiabá, MT-BR. 1. Mestranda em Nutrição e Cirurgia do Programa de Pós-Graduação em Ciências da Saúde da Faculdade de Ciências Médicas da Universidade Federal do Mato Grosso, MT- BR. 2. Professora Assistente do Departamento de Alimentação e Nutrição da Faculdade de Nutrição da Universidade Federal do Mato Grosso, MT- BR. 3. Professor Titular do Departamento de Clínica Cirúrgica da Faculdade de Ciências Médicas da Universidade Federal do Mato Grosso, MT- BR. 
de corte transversal com a avaliação de 87 pacientes maiores de 18 anos, de ambos os sexos, candidatos à procedimento cirúrgico de grande porte no trato gastrointestinal, internados na enfermaria de Clínica Cirúrgica desse hospital durante o período de janeiro a dezembro de 2008. Foram excluídos pacientes menores de 18 anos ou que apresentavam edema em alguma das mãos no momento da avaliação.

\section{Avaliação do estado nutricional}

Os pacientes foram submetidos à avaliação nutricional até 48 horas após a admissão hospitalar, sendo utilizados os seguintes parâmetros: 1. avaliação subjetiva global (ASG) ${ }^{11}$; 2. antropometria clássica; 3. parâmetros laboratoriais tradicionais (albumina sérica e linfocitometria); 4. medida da EMAP.

Os pacientes foram classificados pela $\mathrm{ASG}^{11} \mathrm{em}$ eutróficos (ASG-A), risco nutricional ou moderadamente desnutridos (ASG-B) e desnutridos grave (ASG-C). Para fins de análise estatística, os pacientes foram posteriormente divididos em desnutridos (ASG-B e ASG-C) e não desnutridos (ASG-A).

A antropometria clássica constou de medidas de peso atual, peso habitual (história clínica), altura, índice de massa corporal (peso em kg/altura em $\mathrm{cm}^{2}$ - IMC), percentual de perda de peso, circunferência do braço, prega cutânea triciptal, circunferência muscular do braço. Estas medidas foram realizadas de acordo com os métodos classicamente. ${ }^{12,13}$. A classificação do estado nutricional dos pacientes pelo IMC foi feita segundo a Organização Mundial da Saúde $(1995)^{14}$.

O peso atual foi obtido utilizando balança plataforma com capacidade para $150 \mathrm{~kg}$, graduada em $50 \mathrm{~g}$ da marca Kratos $®$ (Embu, Brasil). Peso habitual foi obtido através da informação colhida do paciente através da pergunta: "qual era seu peso habitual antes de ficar doente?". Para a altura utilizou-se estadiômetro acoplado a balança, com variação de $0,1 \mathrm{~cm}$. Os resultados de albumina sérica $(\mathrm{mg} / \mathrm{dL})$ e linfocitometria $\left(\mathrm{mm}^{2}\right)$ foram obtidos por meio dos exames de rotina realizados no hospital do estudo e anexados no prontuário dos pacientes.
A medida da EMAP foi realizada com o paciente sentado, o braço flexionado a aproximadamente $90^{\circ} \mathrm{com}$ o antebraço e a mão apoiada sobre o joelho. Os pacientes foram orientados a ficar com a mão relaxada. Foi utilizado o plicômetro da marca Cescorf® (Porto Alegre, Brasil) exercendo pressão contínua de $10 \mathrm{~g} / \mathrm{mm}^{2}$ para pinçar o músculo adutor no vértice de um triângulo imaginário formado pela extensão do polegar e indicador. O procedimento foi feito na mão dominante e não dominante por três vezes sendo usado a média como medida da EMAP.

\section{Análise estatística}

Estabeleceu-se a ASG como padrão-ouro para a avaliação nutricional. Os pontos de corte para a EMAP de ambas as mãos, em relação ao estado nutricional, foram determinados por meio de curvas ROC (Receiver Operating Characteristic). Foram também determinados a área total sob a curva ROC e os intervalos de confiança (IC 95\%). A partir daí, calculou-se a sensibilidade e especificidade da EMAP para diagnosticar a desnutrição. A correlação entre todos os métodos de avaliação com o MAP foi testada pelo teste de Pearson. Aplicou-se o teste de Kruskal-Wallis seguido de comparações múltiplas pelo teste de MannWhitney para comparar a EMAP com o estado nutricional avaliado pela ASG. Foi estabelecido nível de significância para rejeição da hipótese de nulidade de $5 \%(p<0,05)$. Todas as análises foram desenvolvidas pelo Statistical Package for the Social Sciences (SPSS) for Windows 13.0.

\section{RESULTADOS}

Três pacientes não puderam participar da pesquisa devido a edema nas mãos.

A média de idade foi de 53,8 $\pm 15,9$ anos. A população foi composta de $48,3 \%(n=42)$ de homens e $51,7 \%(n=45)$ de mulheres. As variáveis de avaliação nutricional estudadas estão apresentadas na tabela 1. A medida da EMAP da mão dominante (EMAPD) foi 12,64

Tabela 1 - Dados demográficos, antropométricos e laboratoriais dos 87 pacientes estudados.

\begin{tabular}{|c|c|c|c|c|c|}
\hline & Média & Mediana & Desvio Padrão & Mínimo & Máximo \\
\hline Idade & 53,8 & 55,0 & 15,4 & 18 & 82 \\
\hline Peso atual (kg) & 63,6 & 61,7 & 19,8 & 37,2 & 150 \\
\hline Peso habitual (kg) & 72,0 & 68,0 & 19,2 & 10 & 150 \\
\hline Altura (m) & 1,62 & 1,61 & 0,10 & 1,32 & 1,80 \\
\hline $\operatorname{IMC}\left(\mathrm{kg} / \mathrm{m}^{2}\right)$ & 24,3 & 22,82 & 7,09 & 13,8 & 52,9 \\
\hline$\%$ perda de peso & 11,7 & 9,17 & 10,2 & 0 & 44,2 \\
\hline $\mathrm{CB}(\mathrm{cm})$ & 28,6 & 27,5 & 7,9 & 18,1 & 75,0 \\
\hline $\mathrm{PCT}(\mathrm{mm})$ & 15,0 & 10,5 & 14,8 & 1,6 & 75,0 \\
\hline $\mathrm{CMB}(\mathrm{cm})$ & 23,59 & 23,02 & 4,35 & 17,03 & 51,45 \\
\hline Albumina (g/dL) & 3,55 & 3,7 & 0,58 & 2,0 & 4,4 \\
\hline Linfócitos $\left(\mathrm{mm}^{3}\right)$ & 1863,9 & 1710,0 & 1093,0 & 260,0 & 8100,0 \\
\hline EMAPD (mm) & 12,64 & 12,55 & 3,19 & 5,6 & 23,9 \\
\hline EMAPND (mm) & 12,23 & 11,70 & 2,9 & 7,4 & 23,1 \\
\hline
\end{tabular}

IMC: índice de massa corporal; CB: circunferência do braço; PCT: prega cutânea triciptal; CMB: circunferência muscular do braço; EMAPD: espessura do músculo adutor do polegar da mão dominante; EMAPND: espessura do músculo adutor do polegar da mão não dominante. 
\pm 3,19 mm e da mão não dominante (EMAPND) 12,23 \pm $2,9 \mathrm{~mm}$.

Quanto ao IMC observou-se que 15 pacientes $(17,2 \%)$ encontravam-se com baixo peso (Tabela 2$)$. Segundo a ASG, 11,5\% dos pacientes foram classificados como ASG-A, 29,9\% ASG-B e 58,6\% em ASG-C (Tabela 3).

Em relação ao diagnóstico, 45 (51,7\%) pacientes apresentavam neoplasias. As operações mais frequentemente realizadas foram gastrectomias $(n=18 ; 20,7 \%)$, colectomias ( $n=14 ; 16 \%)$, derivações bilio-digestivas ( $n=9$; $10,3 \%)$ e esofagectomias ( $n=8 ; 9,2 \%)$.

Na figura 1 (a e b), estão representados a curva ROC do EMAP de ambas as mãos e referidas as respectivas

Tabela 2 - Classificação do estado nutricional dos 87 pacientes estudados de acordo com o índice de massa corporal segundo OMS, 1995.

\begin{tabular}{lll}
\hline Classificação & N & $(\%)$ \\
\hline Baixo peso & 15 & $(17,2 \%)$ \\
Eutrofia & 37 & $(42,5 \%)$ \\
Sobrepeso/obesidade & 35 & $(40,2 \%)$ \\
\hline
\end{tabular}

Tabela 3 - Classificação do estado nutricional dos 87 pacientes estudados de acordo com a avaliação subjetiva global.

\begin{tabular}{ll}
\hline Classificação & N (\%) \\
\hline A - Eutrofia & $10(11,5 \%)$ \\
B - Risco nutricional ou desnutrição moderada & $26(29,9 \%)$ \\
C - Desnutrição grave & $51(58,6 \%)$ \\
\hline
\end{tabular}

a

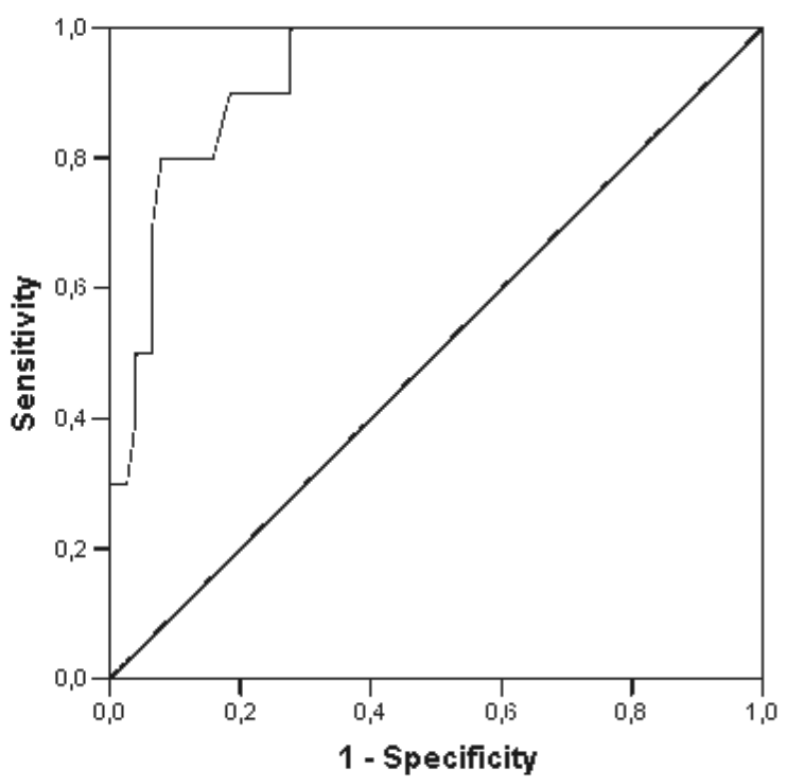

áreas sob a curva ROC para população estudada. O melhor ponto de corte da EMAP da mão dominante para o desfecho desnutrição foi 13,4 mm e para a EMAP da mão não dominante foi de $13,1 \mathrm{~mm}$

Através dos pontos de corte estabelecidos pela curva ROC, encontrou-se sensibilidade de $72,37 \%$ para EMAP da mão dominante e $77,33 \%$ para a da mão não dominante. Já a especificidade encontrada foi de $100 \%$ para ambas as mãos.

Os resultados das diversas técnicas utilizadas para avaliação nutricional dos pacientes segundo o ponto de corte para EMAP dominante e não dominante estão apresentados na tabela 4.

Todas as medidas antropométricas clássicas se correlacionaram de modo significativo com a EMAP dominante e não dominante (Figura 2).

Os valores encontrados para EMAPD e EMAPND entre pacientes considerados nutridos (ASG-A) foram significativamente maiores $(P<0.001)$ daqueles vistos para desnutridos leves (ASG-B) e nestes, os valores também foram maiores $(P<0,05)$ quando comparados aos desnutridos graves (ASG-C).

\section{DISCUSSÃO}

Os achados deste estudo mostram que a EMAP é um método confiável para avaliação do estado nutricional de pacientes cirúrgicos. Não só houve correlação com outros parâmetros antropométricos como também boa sensibilidade e principalmente especificidade. Além disso, quando se compara os resultados desse método com o padrão-ouro de avaliação nutricional, recomendado pela American Society for Parenteral and Enteral Nutrition (ASPEN) ${ }^{15}$, houve também boa correlação. A análise global desses re-

b

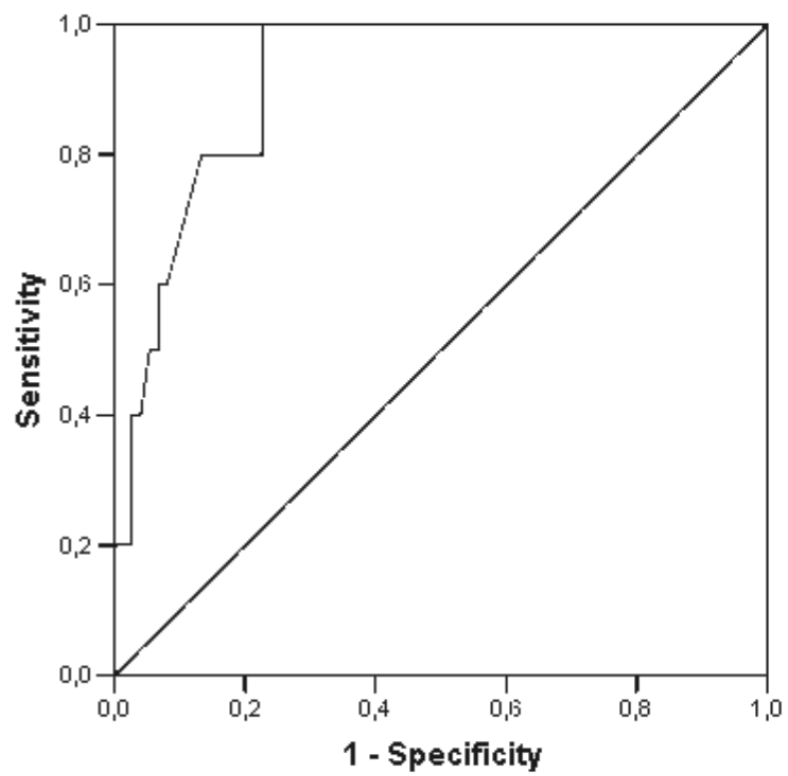

a - Espessura do músculo adutor do polegar da mão dominante (área sob a curva: 0,93 (IC95\%0,86-0,99)); b - Espessura do músculo adutor do polegar da mão não dominante (área sob a curva: 0,92 (IC95\%0,85-0,98)).

Figura 1 - Relação espessura do músculo adutor do polegar e desnutrição dos pacientes estudados. 


\section{EMAPD}

$I M C$

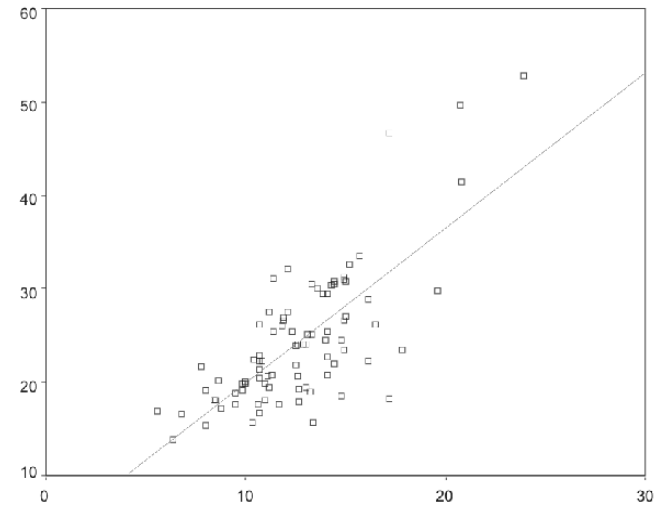

$C M B$

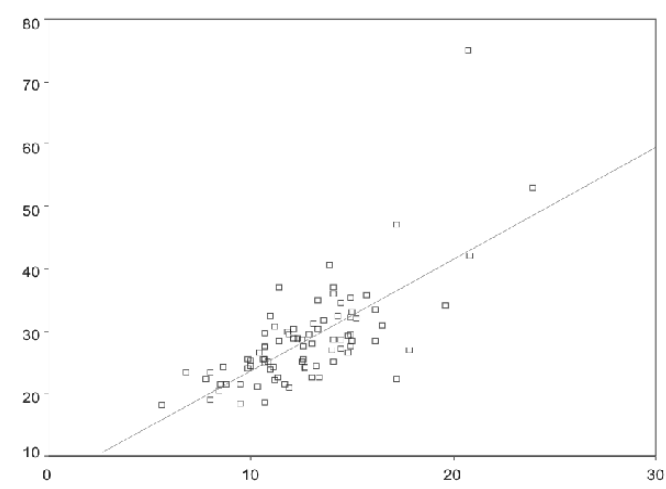

PCT

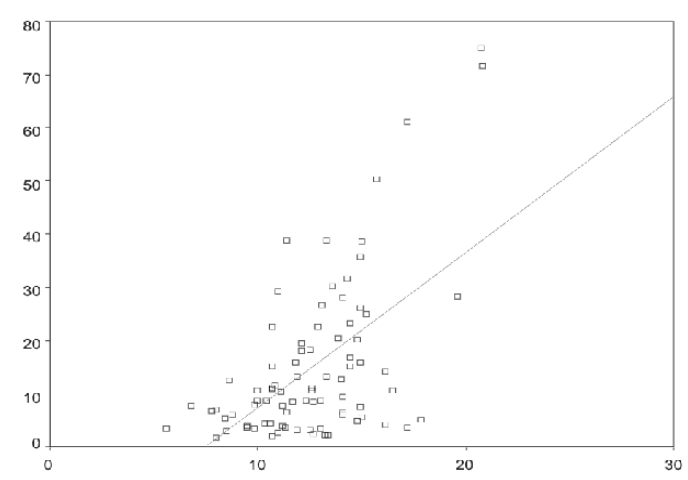

$C M B$

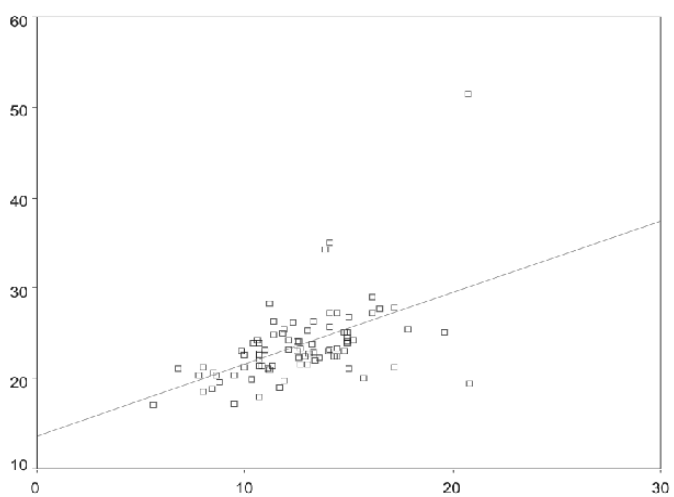

\section{EMAPND}
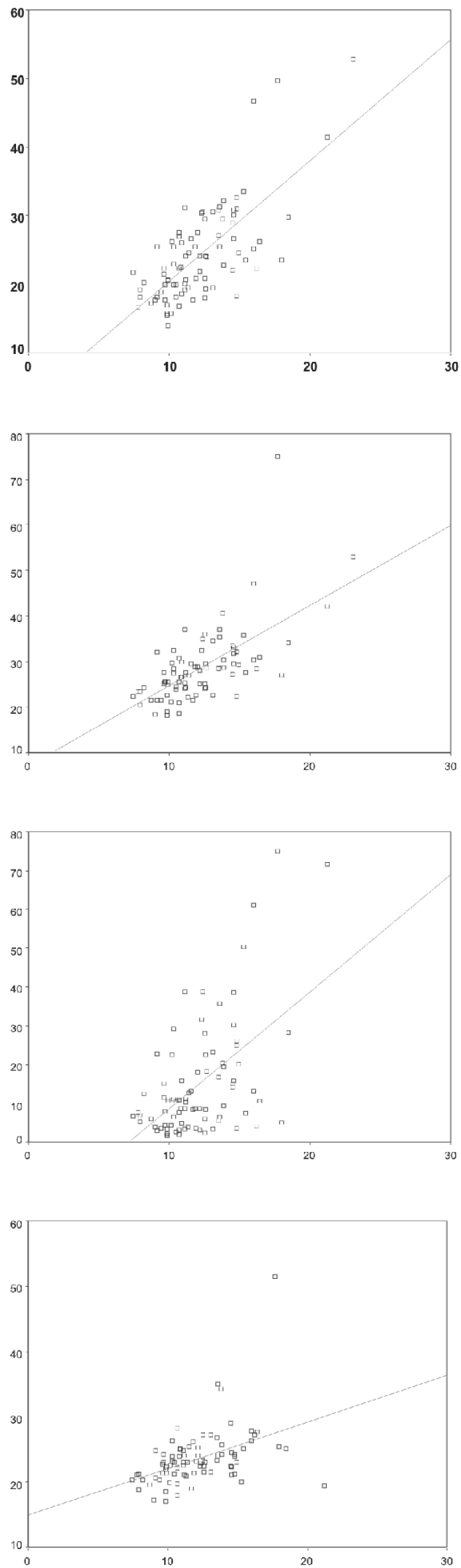

IMC: índice de massa corporal; CMB: circunferência muscular do braço; PCT: prega cutânea triciptal; CMB: circunferência muscular do braço; EMAPD: espessura do músculo adutor do polegar da mão dominante; EMAPND: espessura do músculo adutor do polegar da mão não dominante.

Figura 2 - Correlações da espessura do músculo adutor do polegar de ambas as mãos com várias medidas antropométricas. $P<0,05$ em todas as correlações. 
Tabela 4 - Resultados das medidas antropométricas e laboratoriais dos pacientes estudados de acordo com o estado nutricional determinado pela espessura do músculo adutor do polegar (EMAP) das duas mãos.

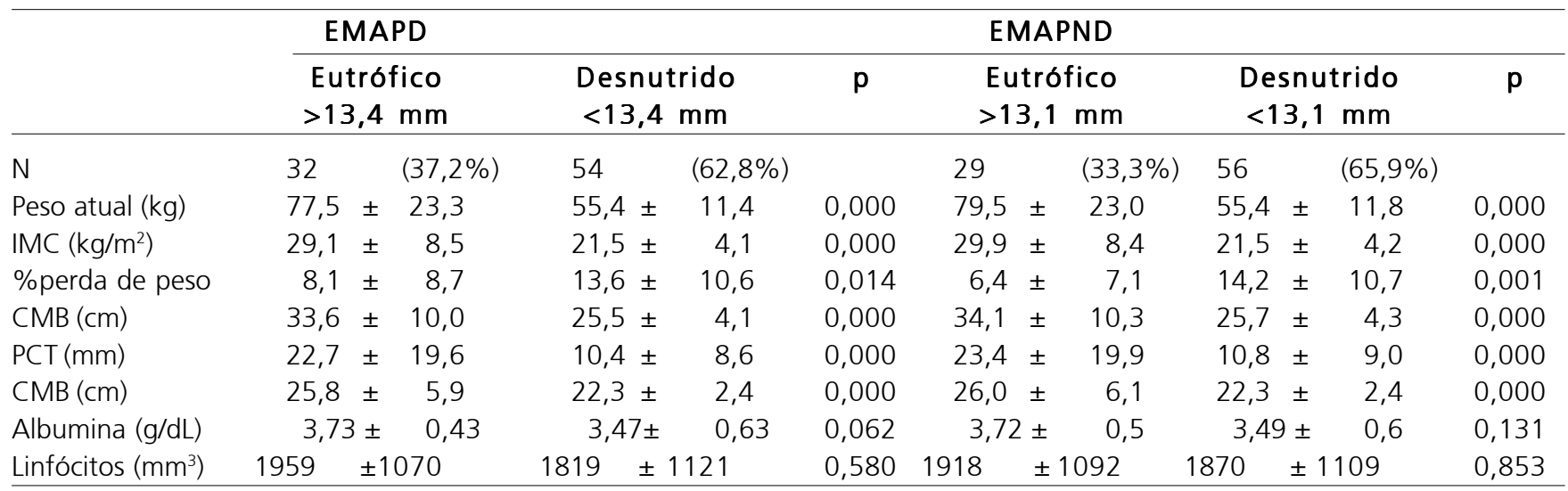

IMC: índice de massa corporal; CMB: circunferência muscular do braço; PCT: prega cutânea triciptal; CMB: circunferência muscular do braço, EMAPD: espessura do músculo adutor do polegar da mão dominante; EMAPND: espessura do músculo adutor do polegar da mão não dominante. Os dados estão apresentados como média \pm desvio-padrão.

sultados sugere que esse novo método, de fácil execução e baixo custo, transmite segurança na avaliação do estado nutricional e por isso pode ser usado na prática clínica em pacientes cirúrgicos.

A avaliação nutricional imediata, logo na admissão do paciente, se faz necessária para que um plano nutricional precoce possa ser instituído, objetivando principalmente identificar os pacientes desnutridos ou em risco de desenvolver, para que não deixem de receber intervenção nutricional adequada e imediata ${ }^{16}$. Nesse sentido, a avaliação nutricional através da EMAP de ambas as mãos apresentou especificidade de $100 \%$, conferindo ótima cobertura.

Como esperado, as médias encontradas da EMAP, de ambas as mãos, foram menores que as encontradas por Budziareck, et al. ${ }^{9}$ em pessoas saudáveis uma vez que a doença, a desnutrição e a diminuição da atividade laboral levam a perda protéica, consequentemente, à depleção muscular ${ }^{17}$. Entretanto, em pacientes internados em clínica médica ${ }^{10}$, a média da EMAP também foi menor que esta, em pacientes cirúrgicos. No entanto os presentes achados foram maiores que os encontrados por Lameu, et al. ${ }^{7}$ em estudantes, técnicos e médicos de um hospital universitário. Essa diferença talvez se deva ao tipo de paciente (cirúrgico ou clínico), tipo de profissão e tipo de aparelho utilizado.

No presente estudo, o percentual de pacientes desnutridos pela ASG $(88,5 \%)$ foi maior que os encontrados em vários outros estudos multicêntricos. Estudo brasileiro (IBRANUTRI) ${ }^{2}$ e latinoamericano ${ }^{18}$ encontrou que 48,1 e $50,2 \%$ respectivamente dos pacientes apresentavam algum grau de desnutrição. Esse resultado é compreensível, uma vez que a amostra estudada constitui de pacientes candidatos a procedimento cirúrgico de grande porte e mais da metade da amostra apresentava neoplasia. Outro enfoque importante é que o IMC subestima o percentual de desnutridos em pacientes hospitalizados ${ }^{19}$, já que por esse método apenas 17,2\% dos pacientes foram considerados desnutridos.

Não houve associação entre EMAP e exames laboratoriais. Entretanto, ela se correlacionou com todas as clássicas medidas antropométricas. Isso se coaduna com a natureza deste exame, visto que ela não deixa de ser uma medida antropométrica. Os grandes pontos positivos do exame em tela são a sua fácil condução, a rapidez dos resultados, os seus custos e finalmente, sua boa correlação com outras medidas antropométricas. Realmente, os poucos trabalhos que antecederam ao atual, já mostravam resultados semelhantes a este.

Além disso, outra vantagem do método é que como o músculo adutor do polegar é plano e situado entre duas estruturas ósseas, possui referência anatômica bem definida ${ }^{7}$, e a avaliação do seu tamanho é direta, quer dizer, não precisa fazer equação para estimar seu tamanho real como é o caso da circunferência muscular do braço.

Outro ponto positivo para este trabalho é que ele representa a primeira avaliação da EMAP em doentes cirúrgicos. De acordo com os resultados encontrados, esta técnica pode ser perfeitamente usada para a avaliação do estado nutricional em pacientes cirúrgicos. Estudos posteriores para avaliação do prognóstico desta medida em cirurgia devem ser realizados para melhor conhecimento do alcance deste novo método de avaliação nutricional.

Em conclusão, a espessura do músculo adutor do polegar é um método de fácil execução, baixo custo, confiável e transmite segurança na avaliação do estado nutricional, podendo ser usado na prática clínica em pacientes cirúrgicos. 
Objective: Investigate whether the right adductor pollicis muscle thickness is a reliable method to evaluate the nutritional status of surgical patients and whether it correlates or not correlate to other anthropometric, biochemical, and clinical parameters. Methods: Cross-sectional study evaluating 87 patients candidates to major operations of the gastrointestinal tract. All were submitted to global subjective evaluation, traditional anthropometry (arm circumpherence; triceps skin fold; and arm muscle circumpherence, serum albumin, lymphocytes and measurement of in both hands. Results: The mean right adductor pollicis muscle thickness in dominant hand was 12,64 $\pm 3,19 \mathrm{~mm}$ and in non-dominant hand (EMAPND) 12,23 $\pm 2,9 \mathrm{~mm}$. Sensitivity was $72,37 \%$ for left adductor pollicis muscle and 77,33\% for left adductor pollicis muscle thickness being the cut-offs given by the ROC curve $(13,4$ and $13,1 \mathrm{~mm}$ respectively). Specificity was $100 \%$ for both hands. There was a significantly correlation $(P<0.01)$ for right adductor pollicis muscle thickness with all anthropometric parameters. The mean value for normal individuals was significantly greater $(P<0.001)$ than for mild malnourished which was greater $(p<0.05)$ when compared to patients with severe malnutrition. Conclusion: right adductor pollicis muscle thickness is a low-cost, reliable, and trustworthy method to assess nutritional status that can be easily and safety used in clinical practice for surgical patients.

Key words: Nutritional assessment. Surgery. Malnutrition. Anthropometry.

\section{REFERENCIAS}

1. Barbosa-Silva MCG, Barros AJD. Avaliação nutricional subjetiva. Parte 1 - Revisão de sua validade após duas décadas de uso. Arq Gastroenterol. 2002; 39(3):181-7

2. Waitzberg DL, Caiaffa WT, Correia MI. Hospital malnutrition: the Brazilian national survey (IBRANUTRI): a study of 4000 patients. Nutrition. 2001: 17(7-8):573-80.

3. Silva MCGB. Avaliação subjetiva global. In: Waitzberg DL. Nutrição oral, enteral e parenteral na prática clínica. $3^{a}$ Ed. São Paulo: Atheneu; 2000. p. 241-52.

4. Correia MI, Waitzberg DL. The impact of malnutrition on morbidity, mortality, length of hospital stay and costs evaluated through a multivariate model analysis. Clin Nutr. 2003; 22(3):235-9.

5. Waitzberg DI, Ferrini, MT. Avaliação nutricional. In: Waitzberg DL. Nutrição enteral e parenteral na prática clínica. $2^{a}$ ed. Rio de Janeiro: Atheneu; 1995. p. 127-35.

6. Teixeira LC, Mello ED, Beghetto MG, Luft VC. A competência dos profissionais em identificar a desnutrição hospitalar. Rev Bras Nutr Clin. 2003; 18(4):173-7.

7. Lameu EB, Gerude MF, Campos AC, Luiz RR. The thickness of the adductor pollicis muscle reflects the muscle compartment and may be used as a new anthropometric parameter for nutritional assessment. Curr Opin Clin Nutr Metab Care. 2004; 7(3):293-301.

8. Lameu EB, Gerude MF, Corrêa RC, Lima KA. Adductor pollicis muscle: a new anthropometric parameter. Rev Hosp Clin Fac Med São Paulo. 2004; 59(2):57-62.

9. Budziareck MB, Pureza Duarte RR, Barbosa-Silva MC. Reference values and determinants for handgrip strength in healthy subjects. Clin Nutr. 2008; 27(3):357-62. Epub 2008 May 2.

10. Andrade PV, Lameu EB. Espessura do músculo adutor do polegar: um novo indicador prognóstico em pacientes clínicos. Rev Bras Nutr Clin. 2007; 22(1):28-35.

11. Detsky AS, McLaughlin JR, Baker JP, Johnston N, Whittaker S, Mendelson RA, Jeejeebhoy KN. What is subjective global assessment of nutritional status? JPEN J Parenter Enteral Nutr. 1987; 11(1):8-13.

12. Lohaman TG, Roche AF, Matorell R. Anthropometric standardization reference manual. Abridged edition. Illinois: Human Kinetics Books; 1991.
13. Blackburn GL, Benotti PN, Bistrian BR, Bothe A, Maini BS, Schlamm HT, Smith MF. Nutritional assessment and treatment of hospital malnutrition. Infusionsther Klin Ernahr. 1979; 6(4):238-50.

14. Organização Mundial de Saúde - OMS. El Estado Físico: Uso e interpretación de la antropometria. OMS Serie de Informes Técnicos 854. Genebra: OMS; 1995.

15. ASPEN Board of Directors and the Clinical Guidelines Task Force. Guidelines for the use of parenteral and enteral nutrition in adult and pediatric patients. JPEN J Parenter Enteral Nutr. 2002; 26(1 Suppl):1SA-138SA. Erratum in: JPEN J Parenter Enteral Nutr. 2002; 26(2): 144.

16. Aguilar-Nascimento JE, Caporossi C, Salomão AB. Acerto: acelerando a recuperação total pós-operatória. Cuiabá: EdUFMT; 2009.

17. Humphreys J, de la Maza P, Hirsch S, Barrera G, Gattas V, Bunout D. Muscle strength as a predictor of loss of functional status in hospitalized patients. Nutrition. 2002; 18(7-8):616-20.

18. Correia MI, Campos AC; ELAN Cooperative Study. Prevalence of hospital malnutrition in Latin America: the multicenter ELAN study. Nutrition. 2003; 19(10):823-5.

19. Dock-Nascimento DD, Aguilar-Nascimento JE, Costa HCBAL, Vale HV, Gava MM. Precisão de métodos de estimativa do peso e altura na avaliação do estado nutricional de pacientes com câncer. Rev Bras Nutr Clin. 2006; 21(2):111-6.

Recebido em 17/11/2008

Aceito para publicação em 21/01/2009

Conflito de Interesse: nenhum.

Fonte de financiamento: nenhuma

\section{Como citar este artigo:}

Bragagnolo R, Caporossi FS, Dock-Nascimento DB, Aguilar-Nascimento JE. Espessura do músculo adutor do polegar: um método rápido e confiável na avaliação nutricional de pacientes cirúrgicos. Rev Col Bras Cir. [periódico na Internet] 2009; 36(4). Disponível em URL: http:// www.scielo.br/rcbc

Endereço para correspondência:

José Eduardo de Aguilar-Nascimento

E-mail: aguilar@terra.com.br 